\title{
Relación costo-efectividad
}

\section{de las intervenciones preventivas contra}

\section{el cáncer cervical en mujeres mexicanas}

\author{
Cristina Gutiérrez-Delgado, PhD en Act, (I) Camilo Báez-Mendoza,Act, ${ }^{(1)}$ \\ Eduardo González-Pier, PhD en Econ, ${ }^{(1)}$ Alejandra Prieto de la Rosa, Soc, (2) Renee Witlen, BA. ${ }^{(3)}$
}

\author{
Gutiérrez-Delgado C, Báez-Mendoza C, \\ González-Pier E, Prieto-De la Rosa A, Witlen R. \\ Relación costo-efectividad \\ de las intervenciones preventivas contra \\ el cáncer cervical en mujeres mexicanas. \\ Salud Publica Mex 2008;50:107-I 18.
}

\begin{abstract}
Resumen
Objetivo. Realizar un análisis de la relación costo-efectividad generalizada (ACEG) para la vacuna anti-VPH, el tamiz por captura de híbridos $(\mathrm{CH})$ y el tamiz por Papanicolaou en el caso mexicano. Material y métodos. Entre abril y agosto de 2007 se desarrolló en México un ACEG de las intervenciones relacionadas con 10 posibles escenarios en un modelo markoviano, bajo la perspectiva del sector público como pagador. Resultados. Los escenarios con cobertura universal muestran un cociente costo-efectividad por AVISA ganado para el Papanicolaou en mujeres de 25 a 64 años de 16678 pesos, para el tamiz por $\mathrm{CH}$ en mujeres de 30 a 64 años de 17285 pesos y para la vacuna en niñas de 12 años de 84008 pesos. El financiamiento anual necesario para estas intervenciones es de 62I, 74I y 2255 millones de pesos, respectivamente. Conclusiones. Se sugiere introducir una combinación selectiva de tamices (Papanicolaou y $\mathrm{CH}$ ) y considerar las ventajas comparativas de aplicación en distintas poblaciones y áreas geográficas. De manera complementaria, se aconseja introducir la vacuna con un precio umbral de I8I pesos por dosis, equiparable en términos del costo y la efectividad a la $\mathrm{CH}$.
\end{abstract}

Palabras clave: cáncer cervical; relación costo-efectividad; financiamiento; tamiz cervical; vacuna VPH; México
Gutiérrez-Delgado C, Báez-Mendoza C, González-Pier E, Prieto-De la Rosa A, Witlen R.

Generalized cost-effectiveness of preventive interventions against cervical cancer in Mexican women: Results of a Markov model from the public sector perspective. Salud Publica Mex 2008;50:107-I I8.

\begin{abstract}
Objective. To develop a generalized cost-effectiveness analysis (GCEA) of the HPV vaccine, hybrid capture screening $(\mathrm{HC})$ and Papanicolaou screening (Pap) in the Mexican context. Materials and Methods. From April to August 2007, in Mexico, a GCEA of the interventions was developed for 10 possible scenarios using a Markov model from the public sector perspective as payer. Results. Scenarios considering $80 \%$ coverage show an ACER per DALY averted of $\$ 16678$ pesos for Pap of women between ages 25 and 64, \$17 277 pesos for $\mathrm{HC}$ of women between ages 30 and 64, and $\$ 84008$ pesos for vaccination of 12-year-old girls. Annual financing of $\$ 621, \$ 741$ and $\$ 2255$ million pesos, respectively, is needed for these scenarios. Conclusions. A selective, combined introduction of Pap-HC screening that considers the comparative advantages of application in different populations and geographical areas is suggested.Additionally, it is suggested to introduce the vaccine once a threshold price of $\$ 181$ pesos per dose -when the vaccine becomes equal in terms of costeffectiveness to $\mathrm{HC}$ - has been achieved.
\end{abstract}

Keywords: cervical cancer; cost-effectiveness; financing; cervical screening; HVP vaccine

(I) Unidad de Análisis Económico, Secretaría de Salud. México.

(2) Centro Nacional de Excelencia Tecnológica en Salud, Secretaría de Salud. México.

(3) Escuela de Medicina de Harvard, Universidad de Harvard. Estados Unidos.

Fecha de recibido: 17 de agosto de 2007 - Fecha de aceptado: 21 de enero de 2008 Solicitud de sobretiros: Dra. Cristina Gutiérrez Delgado. Unidad de Análisis Económico. Secretaría de Salud. Paseo de la Reforma 450, piso 14, Col. Juárez. 06600 México DF, México. Correo electrónico: cgutierrezd@salud.gob.mx 
$E_{\text {tritant }}$ México, el cáncer cervicouterino $(\mathrm{CaCu})$ es el más frecuente en mujeres mayores de 25 años y la undécima causa de mortalidad en la población femenina con 4270 defunciones en 2005, equivalentes a una tasa de mortalidad de 8 casos por cada 100000 mujeres. ${ }^{1}$

$\mathrm{El} \mathrm{CaCu}$ es una enfermedad de lenta progresión que se desarrolla como consecuencia de una infección persistente por alguno de los tipos del virus del papiloma humano (VPH) identificados como de alto riesgo y vinculados con el $\mathrm{CaCu}$; éstos ocasionan de forma gradual lesiones epiteliales de bajo o alto grados en la región cervical (displasias) y, en ausencia de tratamiento, evolucionan hacia el $\mathrm{CaCu}$. Dicha transformación tiene lugar en un periodo de 20 a 30 años a partir de la infección (figura 1a). Afortunadamente, el $\mathrm{CaCu}$ es del todo curable si se detecta en etapas tempranas. ${ }^{2-4}$

México ha emitido una norma oficial mexicana (NOM-014-SSA2-1994) "Sobre el control y tratamiento del cáncer cérvico-uterino" que define los lineamientos a seguir, tanto en el programa nacional de tamizaje (PNT$\mathrm{CaCu}$ ) como en el tratamiento de los casos identificados. ${ }^{5}$ El PNT-CaCu instituido a finales de la década de 1970 cobró relevancia y mayor cobertura a partir de 1998. Cada año se realizan en promedio 7.2 millones de tamices anuales mediante citología cervical (Papanicolaou) de forma gratuita, de los cuales una fracción de 1.2\% resulta positiva, ${ }^{6}$ lo cual permite alcanzar cada tres años una cobertura de $80 \%$ de la población femenina de 25 a 64 años. $^{7}$

Pese a los logros alcanzados en la cobertura, el PNT-CaCu representa problemas operativos, entre ellos la baja sensibilidad del Papanicolaou, de tal manera que casi $40 \%$ de los casos, las más de las veces en etapas tempranas, no se reconoce; asimismo, se requiere personal y equipo especializado para asegurar una buena toma y la interpretación de la prueba depende de la capacidad del laboratorista. ${ }^{8}$ Por último, los esfuerzos para aumentar la cobertura del programa de tamizaje han arrojado pocos resultados debido a barreras de acceso por cuestiones geográficas y situaciones de percepción cultural. ${ }^{9}$

La seguridad social absorbe los gastos del tratamiento del $\mathrm{CaCu}$ desde 1943, pero es hasta 2004 cuando éste se financia a través del Seguro Popular de Salud para mujeres sin seguridad social, ya sea mediante el Catálogo Universal de Servicios Esenciales de Salud (CAUSES), en el caso de las displasias, o el Fondo de Protección contra Gastos Catastróficos (FPGC), para etapas más avanzadas. ${ }^{10}$

En décadas recientes se han desarrollado nuevas intervenciones para fortalecer la prevención contra el $\mathrm{CaCu}$. Entre ellas figuran el desarrollo de la vacuna contra los dos tipos más frecuentes del VPH de alto riesgo y la detección de infecciones por el VPH mediante la captura de híbridos $(\mathrm{CH})$. Puesto que la vacuna sólo confiere inmunidad para los tipos de $\mathrm{VPH}$ precursores de $70 \%$ de los casos de $\mathrm{CaCu}$, aun con cobertura universal, resulta necesario continuar con el tamizaje para la identificación de los casos sin protección inmunológica por vacunación. 4,5

En virtud de la situación actual en México para la prevención y tratamiento del $\mathrm{CaCu}$, surgen dos preguntas básicas de política pública para el sector salud: ¿cuál es la combinación de intervenciones que maximiza los resultados en salud ante un presupuesto limitado? y ¿cuál sería el efecto sobre el gasto de la introducción de nuevas tecnologías? Estas preguntas pueden responderse de modo parcial mediante un análisis de costo-efectividad generalizado (ACEG). ${ }^{11}$

Existen trabajos publicados enfocados en el análisis de la efectividad y la relación costo-efectividad de las intervenciones mencionadas. ${ }^{12-20}$ Estos protocolos basados en modelos matemáticos y ensayos clínicos suministran resultados potenciales positivos respecto de la aplicación de dichas intervenciones en el plano poblacional incluso para países de escasos recursos. Entre los hallazgos notificados en las publicaciones destacan los siguientes:

- La efectividad de la vacuna es mayor cuando se aplica a mujeres que no han iniciado vida sexual, por lo que la mayor parte de los trabajos publicados emplea un análisis de cohorte mediante modelos matemáticos en poblaciones de niñas con edades de 10 a 13 años. ${ }^{*}, 13,15,20$

- Existe un modelo matemático según el cual la vacunación para hombres no es efectiva en términos del costo si la cobertura de vacunación contra el VPH es muy alta en la población femenina. ${ }^{20}$

- Los ensayos clínicos indican que la vacuna no ofrece ningún beneficio significativo en mujeres ya infectadas con alguno de los tipos de VPH incluidos en el biológico. ${ }^{12}$

- Sólo existe evidencia de ensayos clínicos que avala la efectividad de la vacuna por cinco años; empero, aún suscita cierta incertidumbre la aplicación de dosis de refuerzo para mantener un porcentaje alto

\footnotetext{
* Hernandez-Avila M, Lazcano-Ponce E, Reynales-Shigematsu L, Rodriguez E, Myers E. Cost-effectiveness analysis of a potential human papillomavirus vaccine (HPV) and its epidemiological and economic impact on prevention and treatment of cervical cancer in Mexico, technical report, 2006. Merk Sharp and Dome, Dosier presentado al Consejo de Salubridad General para inclusión de la vacuna al cuadro básico de medicamentos. Documento no publicado.
} 
a) Historia natural del $\mathrm{CaCu}$ vinculuada con las políticas públicas de prevención y tratamiento

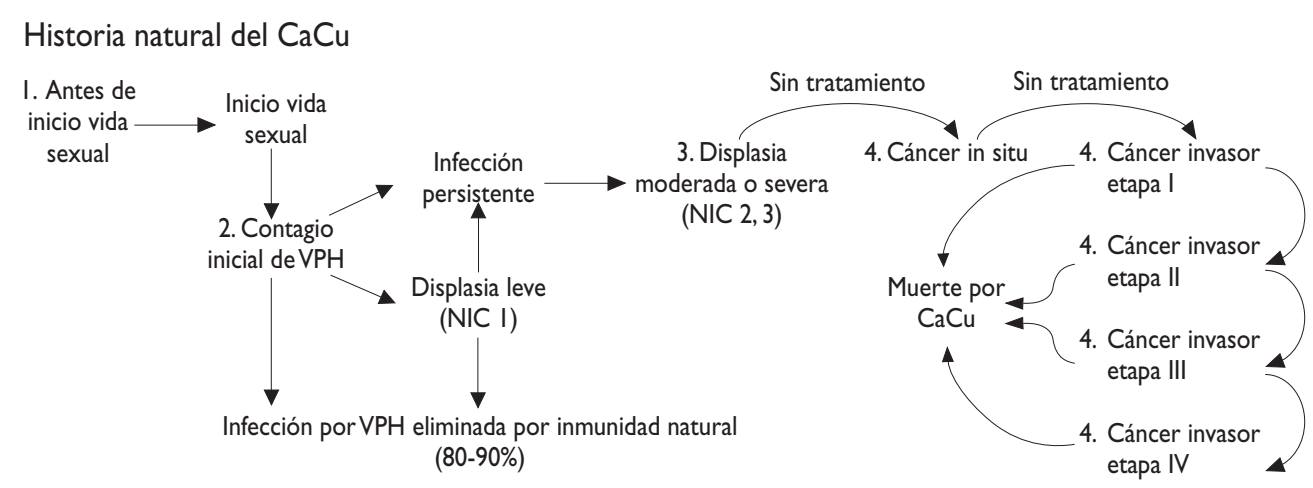

Políticas públicas de salud

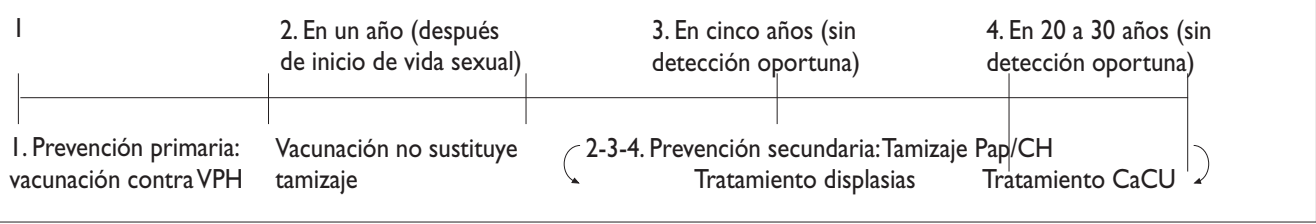

Fuente: Unidad de análisis económico 3,4

b) Modelo markoviano para el ACEG de la historia natural del CaCU en México

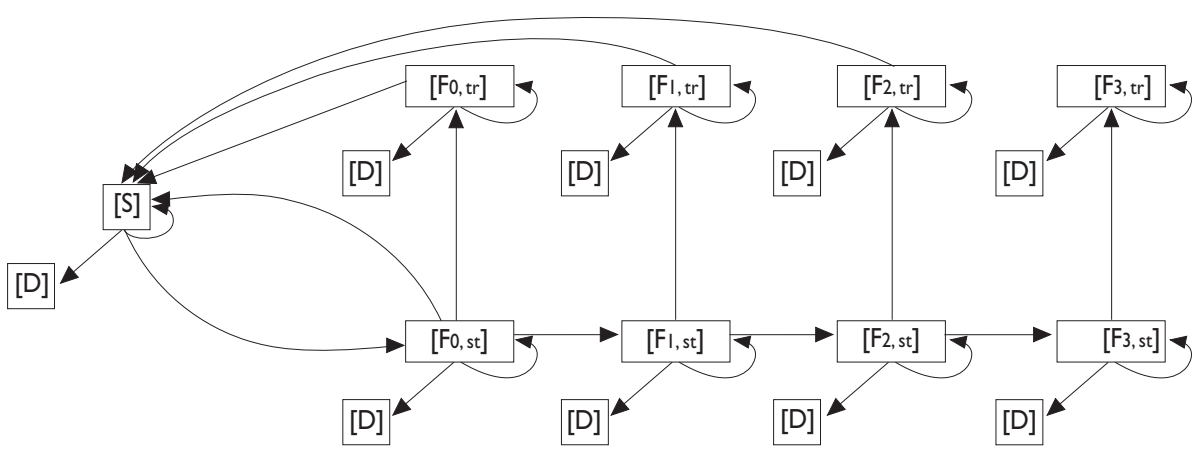

[S]: mujeres susceptibles de infección de cualquier tipo de VPH. [D]: muerte por cualquier causa, incluido el CCU

$[\mathrm{F} 0, \mathrm{st}]$ : mujeres sin tratamiento con lesión intracervical moderada o grave (CIN 2-3)

[F0,tr]: mujeres en tratamiento por lesión intracervical moderada o grave (CIN 2-3)

$[\mathrm{FI}, \mathrm{st}]$ : mujeres sin tratamiento con $\mathrm{CaCu}$ local

$[\mathrm{FI}, \mathrm{tr}]$ : mujeres en tratamiento por $\mathrm{CaCu}$ local

[F2,st]: mujeres sin tratamiento con $\mathrm{CaCu}$ regional

[F2,tr]: mujeres en tratamiento por $\mathrm{CaCu}$ regional

$[\mathrm{F} 3, \mathrm{st}]$ : mujeres sin tratamiento con $\mathrm{CaCu}$ distante

$[\mathrm{F} 3, \mathrm{tr}]$ : mujeres en tratamiento por $\mathrm{CaCu}$ distante

Fuente: Unidad de análisis económico

Figura I. Evolución natural del CaCu y Modelo markoviano para su análisis de COSTO-efEctividad generalizada 
de respuesta inmunitaria durante los 20 a 30 años requeridos para evitar el desarrollo del $\mathrm{CaCu}^{13}$

- El método de tamizaje por $\mathrm{CH}$ es mucho más sensible $(85-87 \%)$ que el tamizaje por Papanicolaou (58.4\%), lo que supone una potencial disminución significativa de falso-negativos..$^{6,21}$

- La toma de muestra para $\mathrm{CH}$ es más sencilla en comparación con el Papanicolaou, ya que no requiere equipo o personal especializado.

- El procesamiento de la muestra por $\mathrm{CH}$ es automatizado, de tal manera que se agilizan el tiempo de obtención y la homogeneidad de los resultados. ${ }^{6}$

En años recientes, la Organización Mundial de la Salud (OMS) y varios países con programas nacionales eficientes de tamizaje por Papanicolaou ${ }^{22-29}$ han publicado recomendaciones respecto de la posible introducción de las intervenciones en el contexto de sus servicios públicos de salud con la intención de determinar el efecto marginal que tendrían en el número de casos potencialmente evitados sobre la morbilidad y la mortalidad. En contraste, México enfoca su interés en identificar intervenciones que robustezcan la prevención y el PNT-CaCu en un contexto de restricción financiera. ${ }^{*}, 30$

A la luz de los antecedentes expuestos, el presente trabajo tiene como objetivo desarrollar un ACEG en el contexto mexicano; la finalidad es determinar la combinación más efectiva en relación con el costo de las intervenciones preventivas mencionadas, desde la perspectiva del sector público como patrocinador.

\section{Material y métodos}

La vacuna contra el VPH se compone de proteínas que forman partículas semejantes al virus del VPH; éstas, de modo conjunto con una sustancia adyuvante, inducen una respuesta inmunitaria. Dos laboratorios farmacéuticos elaboran la vacuna; en este trabajo se analizó el producto de Merck Sharp \& Dohme (MSD), dado que hasta agosto de 2007 sólo éste contaba con la licencia sanitaria en México. Dicha vacuna inmuniza contra los tipos 6 y 11 no vinculados con el $\mathrm{CaCu}$ y 16 y 18 relacionados con él. El esquema sugerido para la vacuna incluye tres dosis de $0.5 \mathrm{ml}$ cada una. La administración de la segunda y tercer dosis se sugiere al segundo y sexto meses después de la primera. La vacuna se vende en jeringas precarga-

\footnotetext{
* Centro Nacional de Equidad de Género y Salud Reproductiva. Propuesta de mejora del programa de prevención y control del $\mathrm{CaCu}$. Secretaría de Salud, 2007. Documento no publicado.
}

das y requiere la intervención de personal capacitado para aplicar inyecciones intramusculares. ${ }^{*}$

La prueba de $\mathrm{CH}$, que produce el laboratorio Digene Diagnostics, utiliza la tecnología Hybrid Capture 2, cuya sensibilidad hace posible reconocer la presencia de 18 tipos de VPH en especímenes cervicales. $\mathrm{La} \mathrm{CH}$ puede diferenciar entre VPH de bajo riesgo no vinculado con $\mathrm{CaCu}$ (tipos 6, 11, 42, 43y 44) y VPH de alto riesgo relacionado con $\mathrm{CaCu}$ (tipos 16, 18, 31, 33, 35, 39, 45, $51,52,56,58,59$ y 68$)$, pero no puede determinar el tipo específico de VPH presente en la muestra analizada. Para la $\mathrm{CH}$ es preciso tomar una muestra de células de la pared cervical mediante un hisopo que se coloca en un tubo con fijador, el cual se procesa mediante equipo automatizado. ${ }^{30} \mathrm{La}$ toma pueden realizarla el personal de salud o la propia paciente. ${ }^{31}$ Se sugiere la aplicación de la prueba a partir de los 30 años de edad. Si el resultado es negativo, se aconseja repetir la prueba en un periodo de 2 a 5 años. Cuando el resultado es positivo está indicada una prueba de Papanicolaou para determinar la existencia de lesión antes de remitir a la mujer a la colposcopía. ${ }^{32}$

Para la prueba de Papanicolaou es necesario que personal capacitado tome una muestra de células de la pared cervical con un espejo vaginal y un hisopo o cepillo cervical. La muestra se fija a una laminilla de vidrio y se envía al laboratorio; allí, un citotecnólogo, mediante un microscopio, observa las células y determina si existe alguna anormalidad, en cuyo caso lo verifica un pató$\operatorname{logo.}{ }^{33}$ La NOM vigente especifica que el Papanicolaou se aplique a las mujeres con edades de 25 a 64 años de forma anual; si la paciente presenta dos tomas anuales negativas consecutivas entonces las siguientes deben efectuarse cada tres años. ${ }^{5}$

El ACEG de las intervenciones se desarrolló entre abril y agosto de 2007 en México mediante un modelo markoviano que simula la evolución natural de la infección por VPH y su interacción con los diversos niveles de prevención y atención para la población femenina mexicana, según los lineamientos de la CONAPO de $2006^{34}$ (figura 1b). Las intervenciones preventivas dividen a la población en las cohortes de niñas a vacunar (prevención primaria), complementadas en varios escenarios con el tamizaje (prevención secundaria), y las cohortes de mujeres no vacunadas con tamizaje solo.

El modelo desarrollado utilizó el marco de los modelos ACEG que propuso la OMS en su proyecto

\footnotetext{
* Merck Sharp \& Dohme. Vacuna recombinante tetravalente contra el virus del papiloma humano tipos 6, 11, 16, 18, 2006. Documento no publicado.
} 
WHO-CHOICE., ${ }^{*}$ El ACEG compara intervenciones contra la opción de no hacer nada (escenario nulo), de tal manera que se resuelven preguntas básicas de eficiencia distributiva de recursos financieros mediante las combinaciones de las intervenciones más efectivas en términos del costo. El modelo calcula los costos por año de vida saludable (AVISA) ganado para cada intervención. En la aplicación del modelo se utilizó el programa Excel para obtener la portabilidad y transparencia requeridas en el proceso de diseminación hacia áreas operativas dentro del sector salud.

El ACEG base considera 20 años de proyección para el uso-financiamiento de las intervenciones y 100 años de proyección para los beneficios en salud; de ese modo se captan los beneficios a largo plazo de la vacuna anti-VPH. ${ }^{11}$ Esta diferenciación en las proyecciones se explica porque las mejores prácticas actuariales sugieren no realizar proyecciones financieras más allá de un plazo de cinco años debido al gran nivel de incertidumbre existente en los costos de la atención médica. ${ }^{35} \mathrm{Sin}$ embargo, dado que el $\mathrm{CaCu}$ presenta una evolución natural de unos 20 a 30 años, se consideró importante aumentar dicho límite para delinear el efecto financiero derivado del periodo mínimo de desarrollo de la enfermedad.

La perspectiva que se aplica en el análisis es la del pagador, representado por el sector público e integrado en México por las instituciones de seguridad social, los servicios estatales de salud, los institutos nacionales y los hospitales federales de referencia. En este análisis sólo se consideran los costos directos derivados de la operación de las intervenciones porque se asume que las intervenciones estudiadas se incorporarán a programas ya establecidos. Los parámetros y supuestos empleados en el modelo se presentan en el cuadro I.

En el caso de la vacuna, luego del análisis de las referencias publicadas ${ }^{13,15,20}$ y el porcentaje por grupo de edad de mujeres con inicio de vida sexual (según la Encuesta Nacional de Salud $2000^{36}$ ), se decidió considerar las cohortes de edad de 12 años para su aplicación. Esta edad se considera como parte de un escenario conservador. Además, se definieron dos niveles de cobertura. Por un lado, $10 \%$ vinculado con la posibilidad de introducir bajo una prueba piloto la vacuna a pobla-

\footnotetext{
* También se consultó un modelo previo desarrollado en 2006 que sigue el marco de los modelos ACEG y es parte de la colaboración entre la Secretaría de Salud y la Harvard Initiative for Global Health para el caso del tratamiento del $\mathrm{CaCu}$. Este modelo se informa en Gonzalez-Pier, Gutierrez-Delgado, Carvalho S, Stevens C, Casey $\mathrm{D}$, Dias J, et al. Priority setting for health interventions in Mexico. Secretaría de Salud-Universidad de Harvard-Instituto Nacional de Salud Pública, 2006. Documento no publicado.
}

ciones en mayor riesgo entre las niñas de 12 años $^{*} \mathrm{y}$, por otro, $80 \%$ correspondiente a la cobertura más alta para la vacunación por hepatitis B en adolescentes informada en la bibliografía, ${ }^{37}$ puesto que no existen datos publicados de inmunización en adolescentes mexicanos.

En el caso del tamiz por $\mathrm{CH}$, se decidió asumir una periodicidad de tres años luego de considerar que este lapso se aproxima más a lo establecido en la NOM vigente, aunque existen publicaciones que analizan periodicidades de 2 a 5 años. ${ }^{16,32}$

En respuesta al objetivo planteado, y a la luz de los supuestos específicos definidos para las intervenciones y las preguntas de política pública expuestas, se desarrollaron los siguientes escenarios:

0. Escenario nulo: no instituir intervenciones de prevención pero sí de tratamiento.

1. Tamizaje con Papanicolaou: lineamientos de la NOM vigente; se asume una cobertura de $80 \%$ de la población femenina de 25 a 64 años cada tres años.

2. Tamizaje con Papanicolaou- $\mathrm{CH}$ : se presupone una cobertura del tamiz de $90 \%$ de la población de 25 a 64 años cada tres años mediante $80 \%$ de Papanicolaou y $10 \%$ de $\mathrm{CH}$, de acuerdo con la periodicidad recomendada en la NOM vigente.

3. Tamizaje por CH: se aplica a la población de 30 a 64 años; la prueba se realiza cada tres años si el resultado es negativo y se considera una cobertura de $80 \%$ de la población objetivo.

4. Plan piloto de vacunación: vacunación a 10\% de las niñas de 12 años según el esquema recomendado (tres dosis).

5. Vacunación universal: inmunización a 80\% de las niñas de 12 años de acuerdo con el esquema recomendado.

6. Plan piloto de vacunación para niñas de 12 años y tamizaje por Papanicolaou para mujeres de 25 a 64 años.

7. Vacunación universal para niñas de 12 años y tamizaje mediante Papanicolaou para pacientes de 25 a 64 años.

8. Plan piloto de vacunación para niñas de 12 años y tamizaje por $\mathrm{CH}$ para mujeres de 30 a 64 años.

9. Vacunación universal para niñas de 12 años y tamizaje por $\mathrm{CH}$ para mujeres de 30 a 64 años.

El ACEG permitirá calcular el efecto financiero de los escenarios expuestos. Los montos previstos pro-

\footnotetext{
* Centro Nacional de Equidad de Género y Salud Reproductiva. Propuesta de mejora del programa de prevención y control del $\mathrm{CaCu}$. Secretaría de Salud, 2007. Documento no publicado.
} 


\section{Cuadro I}

Parámetros del modelo: CAso base y anÁlisis de Sensibilidad (*)

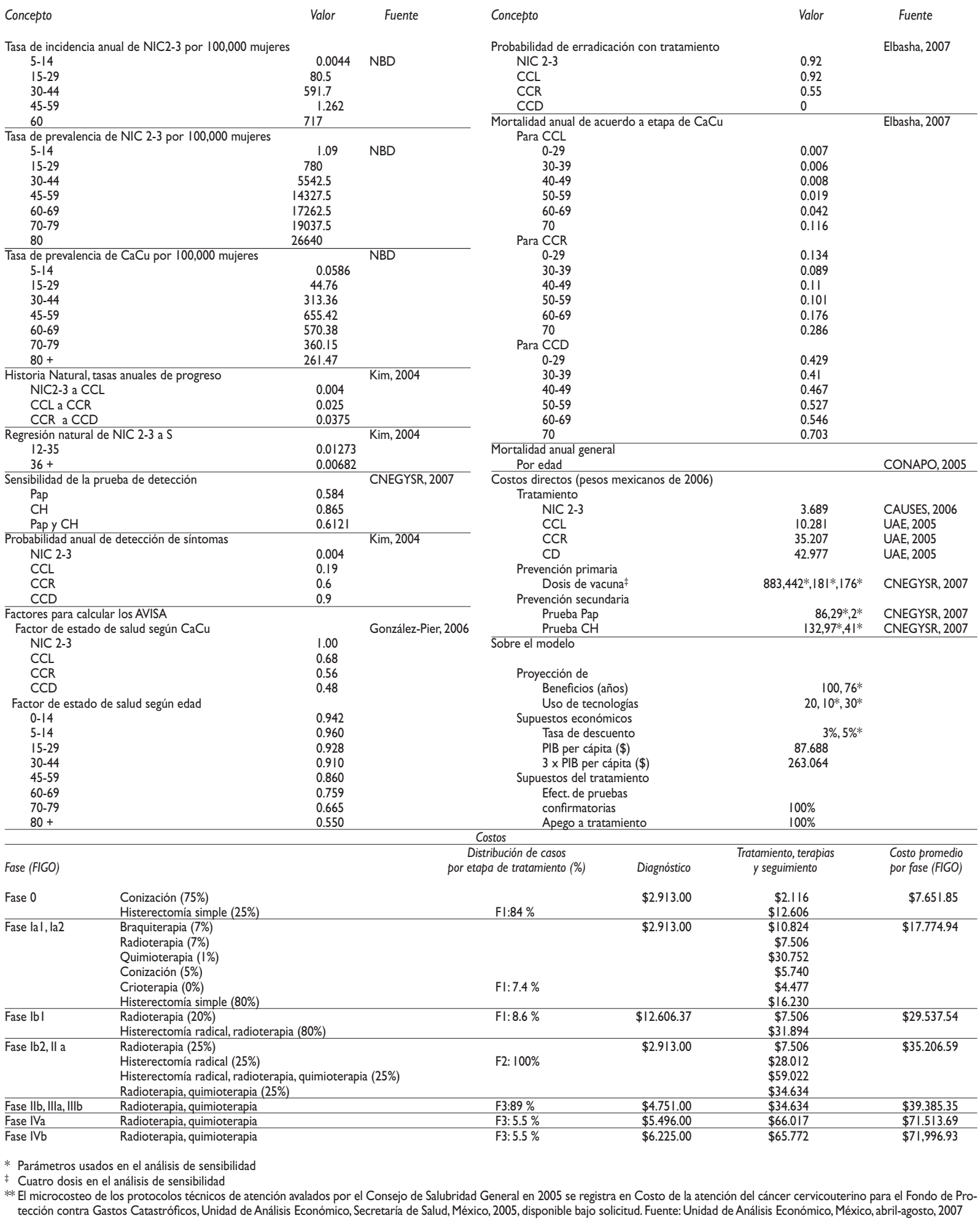

NIC 2-3: neoplasia intraepitelial cervical moderada y grave; CCL: CaCu local; CCR: CaCu regional; CCD: CaCu distante. CNEGYSR: Centro Nacional de Equidad de Género y Salud Reproductiva, Secretaría de Salud; UAE: Unidad de Análisis Económico, Secretaría de Salud; NBD:Mexico: National Burden of Disease, México 2006, Secretaría de Salud-Harvard Initiative for Global Health; CONAPO: Consejo Nacional de Población; Kim 2004, Cost-effectiveness of organized versus opportunistic cervical cytology screening in Hong Kong, Journal of Public Health 26(2) 
porcionarán mayor información acerca de los recursos potencialmente requeridos para cubrir la combinación de intervenciones más efectiva respecto del costo.

Además, dada la complejidad del modelo y la incertidumbre existente en algunos parámetros, se realizará un análisis de sensibilidad. Debido a la escasez de datos, dicho análisis se desarrollará en forma univariada. Los parámetros a estudiar, por ser clave desde la perspectiva financiera para el cumplimiento del objetivo propuesto, son el precio de la vacuna, la aplicación de una dosis de refuerzo, la periodicidad y el precio del tamizaje por $\mathrm{CH}$ y la tasa de descuento.*

\section{Resultados}

Para interpretar los resultados del ACEG se aplica la convención propuesta por la Comisión de Macroeconomía y Salud de la OMS, que considera una intervención como muy efectiva en términos del costo si su razón de costoefectividad promedio (ACER, por sus siglas en inglés) es menor a un producto interno bruto per cápita (PIBpc). Una intervención es efectiva respecto del costo si su ACER fluctúa entre una y tres veces el PIBpc. En 2006 el PIBpc de México fue de 87688 pesos. Los resultados monetarios se expresan en pesos de 2006.

La figura 2 presenta la ruta de expansión (a), el comparativo de los ACER (b) y los resultados más importantes del ACEG (c) para los diversos escenarios analizados. Los resultados indican que el tamizaje por CH es la intervención más efectiva en términos del costo (17 285 pesos por AVISA ganado) después del tamizaje por Papanicolaou (16 678 pesos por AVISA ganado). Los escenarios de tamizaje sin vacuna son más efectivos en cuanto al costo en comparación con los que incluyen vacunación (todos inferiores a 18000 por AVISA ganado). De los escenarios con vacunación, la combinación de vacunación universal y tamizaje por $\mathrm{CH}$ es la más efectiva respecto del costo (31 297 por AVISA ganado). En todos los casos en los que se incluye la vacuna, ésta contribuye con un número discreto de AVISA en comparación con el aporte proveniente de las pruebas de tamiz, si bien su contribución en los costos es muy significativa (figura 2c y cuadro II). La ruta de expansión tecnológica indica que los escenarios a seguir son el tamizaje por Papanicolaou, el tamizaje por $\mathrm{CH}$ y la vacunación universal, los tres con cobertura de $80 \%$ de las respectivas poblaciones objetivo (figura 2a).

\footnotetext{
* En un trabajo futuro se realizará un análisis acerca de la sensibilidad del costo del tratamiento para el $\mathrm{CaCu}$, ya que la falta de datos impide por el momento identificar los límites de variación de los precios de adquisición del sector público.
}

Los resultados del análisis de sensibilidad sobre la vacuna muestran que su precio es la variable más sensible en el modelo (figura 3a). Se observa así que, con objeto de que la vacuna sola logre un resultado casi tan efectivo en términos del costo como el tamizaje por $\mathrm{CH}$, se requiere un precio de 181 pesos por dosis y uno de 176 pesos para compararlo contra el tamizaje por Papanicolaou.

Se considera la aplicación de un refuerzo a los 15 años tras completar el esquema básico, ya que se calcula que en ese año la vacuna alcanza una efectividad de $90 \%$ y se intenta minimizar el número de mujeres susceptibles. Este cálculo se basa en la actualización más reciente del ensayo clínico FUTURE II.* La aplicación de un refuerzo empeora la razón costo-efectividad por arriba de un PIBpc (92 748 contra 84008 con esquema de tres dosis). Al variar la tasa de descuento a $5 \%$ se obtiene un peor resultado para los escenarios en los que se aplica la vacuna (230 281 por AVISA ganado con cobertura de $80 \%$ ).

El análisis de sensibilidad sobre la periodicidad del tamizaje por $\mathrm{CH}$ (figura $3 \mathrm{~b}$ ) muestra que si éste se aplica cada cinco años, entonces se convierte en la intervención más efectiva en cuanto al costo de las opciones analizadas, pero aporta menos AVISA (1 228364 contra 1464587 con aplicación cada tres años). La aplicación de $\mathrm{CH}$ según la NOM vigente es la opción menos efectiva respecto del costo en virtud del costo de la prueba. Los resultados del análisis de sensibilidad sobre el precio de la $\mathrm{CH}$ (figura 3c) muestran que al reducir a 97 pesos esta intervención ya es más efectiva en relación con el costo que el tamiz por Papanicolaou a precio de 86 pesos. Al considerar un precio del Papanicolaou de 29 pesos (obtenido del CAUSES 2006), el tamiz por $\mathrm{CH}$ debe reducir su precio a 40.85 (3.75 dólares) para equipararse en términos de la relación costo-efectividad.

El cuadro II muestra que los montos anuales previstos de los escenarios más efectivos respecto del costo son de 621.8 millones de pesos para el tamizaje por Papanicolaou, 730.8 para el tamizaje por $\mathrm{CH}$ y 2985.3 millones para el tamizaje por $\mathrm{CH}$ y la vacunación universal; la vacuna es el componente más costoso. El cómputo de la población femenina beneficiada en dichos escenarios es de 7.3 millones (mujeres de 25 a 64 años), 5.6 millones (mujeres de 30 a 64 años) y 6.4 millones (niñas de 12 y mujeres de 30 a 64 años), respectivamente. Nótese que los montos a 5 y 20 años siguen un comportamiento poblacional no lineal decreciente.

* Barr E. Updated efficacy data-Gardasil @. HPV vaccines. Merck Research Laboratories, 2007. Documento no publicado. 
(a) Ruta de expansión de los escenarios

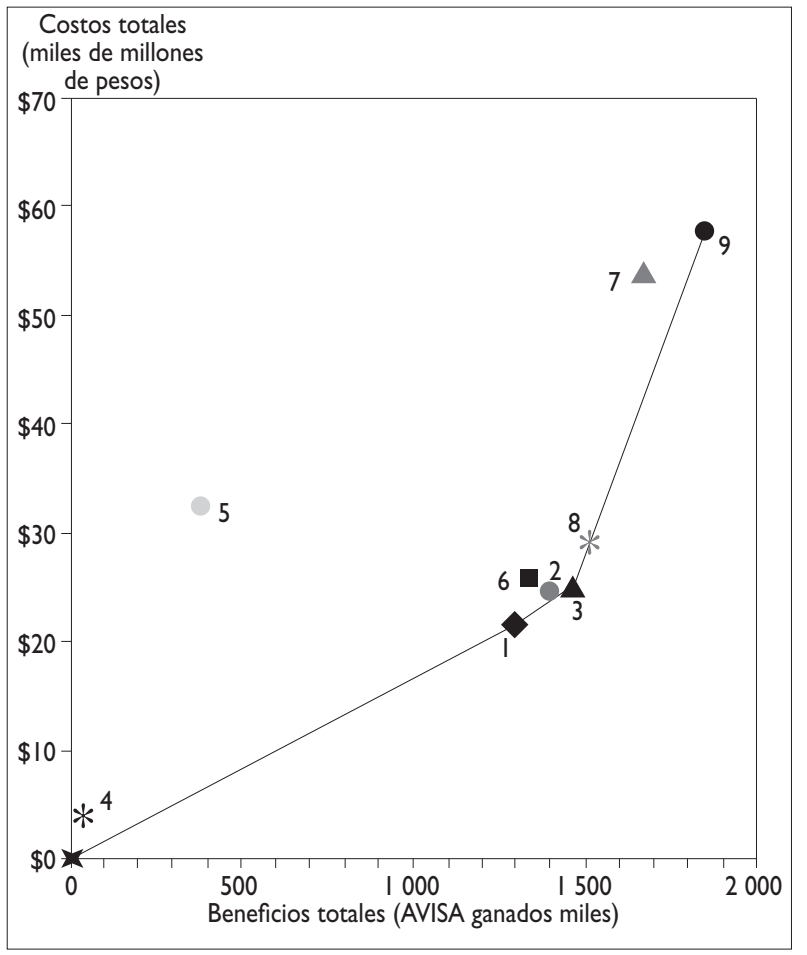

(b) Razón de costo-efectividad promedio

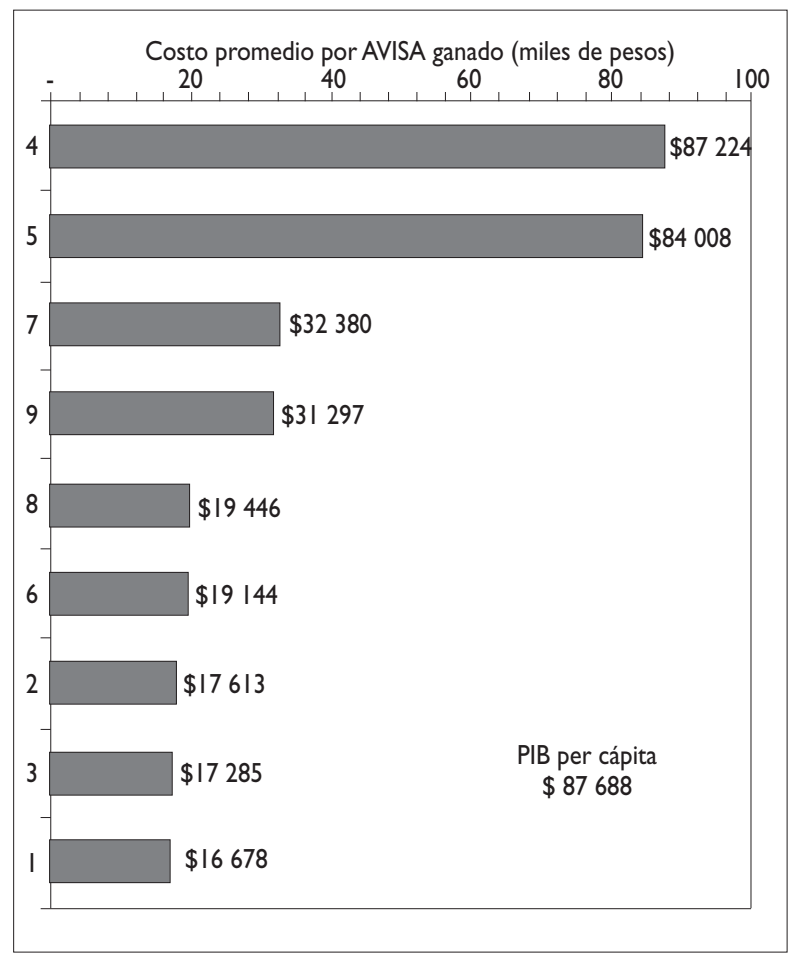

(c) Resumen de resultados del ACEG

\begin{tabular}{|c|c|c|c|c|c|}
\hline \multirow[b]{2}{*}{ No. } & \multirow[b]{2}{*}{ Descripción del escenario } & $\begin{array}{l}\text { Valor presente del costo } \\
20 \text { años para el pagador } \\
\text { (millones de pesos) }\end{array}$ & $\begin{array}{c}\text { Efectividad } \\
\text { (AVISA ganados } \\
\text { en } 100 \text { años) }\end{array}$ & \multirow{2}{*}{$\begin{array}{c}\text { ACER } \\
\text { (pesos por } \\
\text { AVISA ganado) }\end{array}$} & \multirow{2}{*}{$\begin{array}{c}\text { ICER } \\
\text { (pesos por } \\
\text { AVISA ganado) }\end{array}$} \\
\hline & & \multicolumn{2}{|c|}{ Descuento (3\% anual) } & & \\
\hline I & Tamizaje Pap según NOM(3I/05/07) cobertura 80\% & 21.590 & 1.294 .582 & 16.678 & 16.678 \\
\hline 2 & Tamizaje Pap y CH según NOM(3I/05/07) cobertura 90\% & 24.530 & I.392.71। & 17.613 & Dominada \\
\hline 3 & Tamizaje $\mathrm{CH}$ cada tres años cobertura $80 \%$ & 25.316 & 1.464 .587 & 17.285 & 21.914 \\
\hline 4 & Vacunación cobertura $10 \%$ cohorte niñas 12 años & 4.061 & 46.558 & 87.224 & Dominada \\
\hline 5 & Vacunación cobertura $80 \%$ cohorte niñas 12 años & 32.488 & 386.720 & 84.008 & Dominada \\
\hline 6 & Vacunación cobertura $10 \%$ cohorte niñas 12 años y tamizaje Pap cobertura $80 \%$ & 25.644 & 1.339 .558 & 19.144 & Dominada \\
\hline 7 & Vacunación cobertura $80 \%$ cohorte niñas I2 años y tamizaje Pap cobertura $80 \%$ & 54.020 & 1.668 .308 & 32.380 & Dominada \\
\hline 8 & Vacunación cobertura $10 \%$ cohorte niñas 12 años y tamizaje $\mathrm{CH}$ cada 3 años cobertura $80 \%$ & 29.374 & 1.510 .530 & 19.446 & Dominada \\
\hline 9 & Vacunación cobertura $80 \%$ cohorte niñas 12 años y tamizaje $\mathrm{CH}$ cada 3 años cobertura $80 \%$ & 57.783 & 1.846 .274 & 31.297 & 85.061 \\
\hline
\end{tabular}

AVISA: años de vida saludable;ACER: razón costo-efectividad promedio; ICER: razón costo-efectividad incremental.

Dominada: intervención con mayor costo pero menor beneficio en comparación con otras intervenciones analizadas.

Fuente: Unidad de Análisis Económico, México, abril-agosto 2007.

\section{Figura 2. Resultados del análisis de la relación costo-efectividad generalizada del CaCu en México}

En cuanto al gasto en tratamiento, se observa que en todos los escenarios con tamiz el número previsto de tratamientos de displasias es mayor que el número calculado de tratamientos por $\mathrm{CaCu}$ y que es mayor el número de displasias detectadas al utilizar el tamizaje por $\mathrm{CH}$ dada su mayor sensibilidad.

\section{Discusión}

En el presente trabajo se realizó un ACEG de tres intervenciones preventivas contra el $\mathrm{CaCu}$ mediante un modelo markoviano y con base en el marco WHO-CHOICE. ${ }^{11}$ El ACEG permitió determinar la 


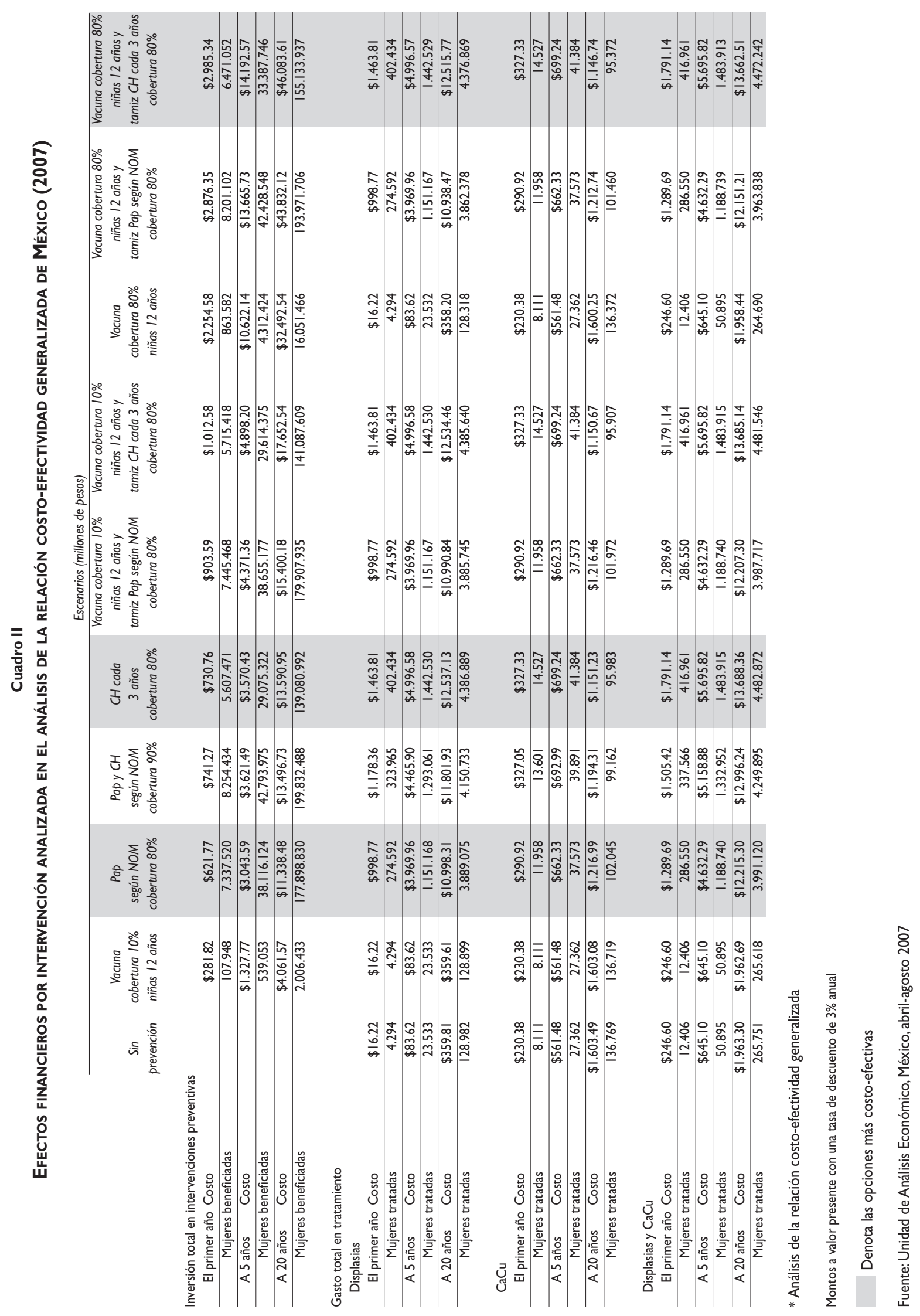


a) Variación en precio para la vacuna VPH

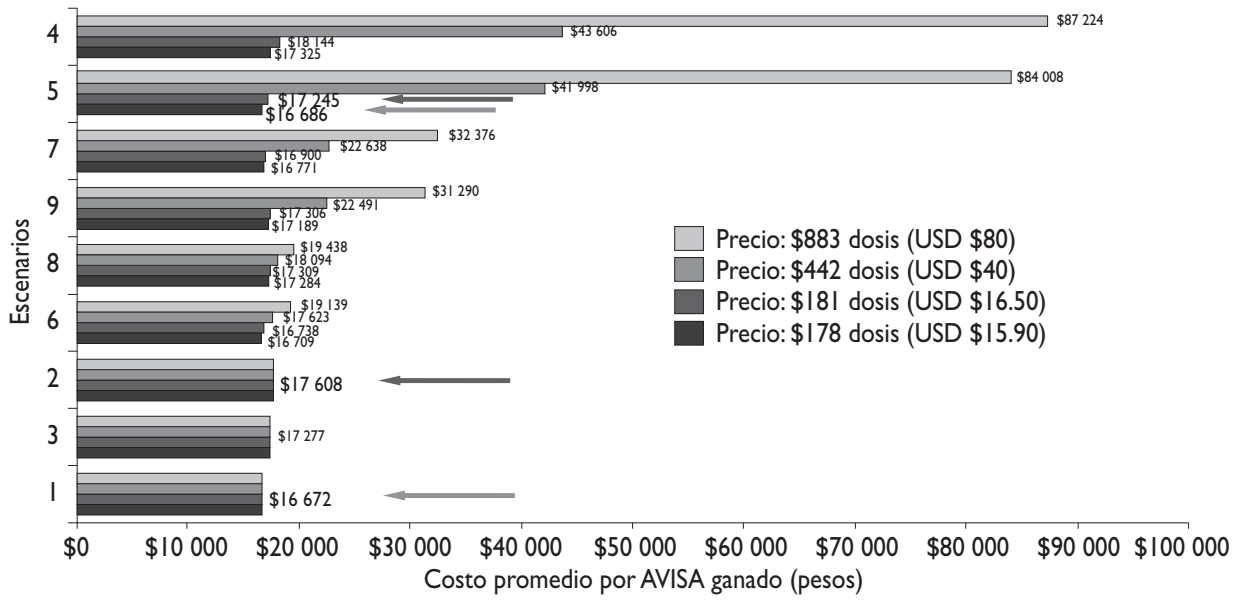

b) Variación en frecuencia de aplicación para el tamiz por $\mathrm{CH}$

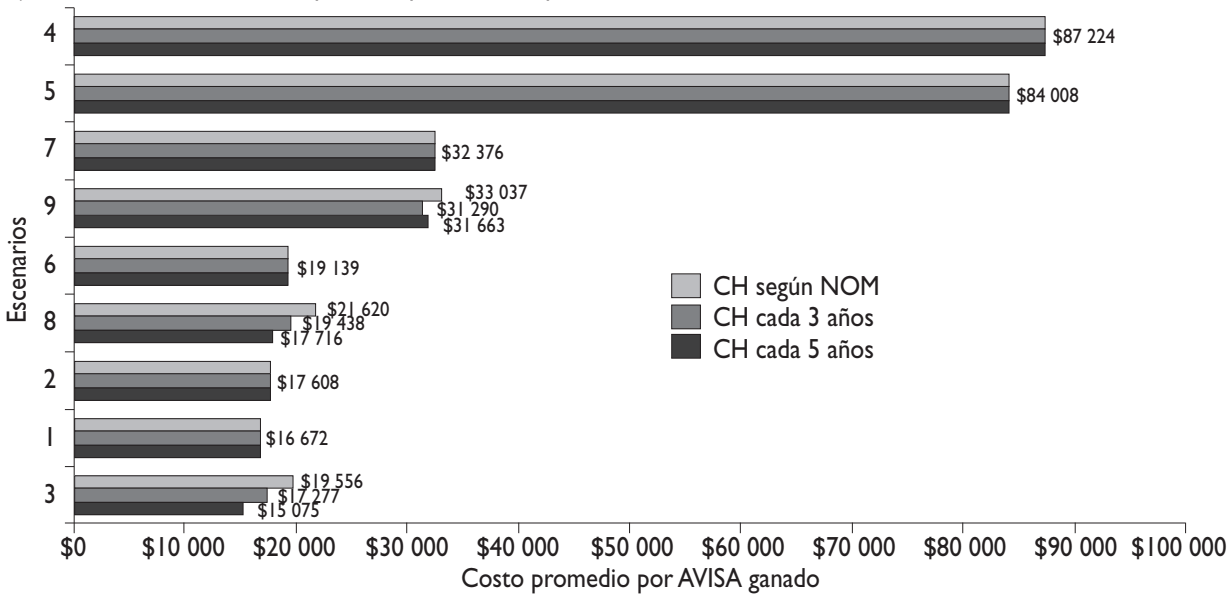

c) Variación en precio para el tamiz por $\mathrm{CH}$

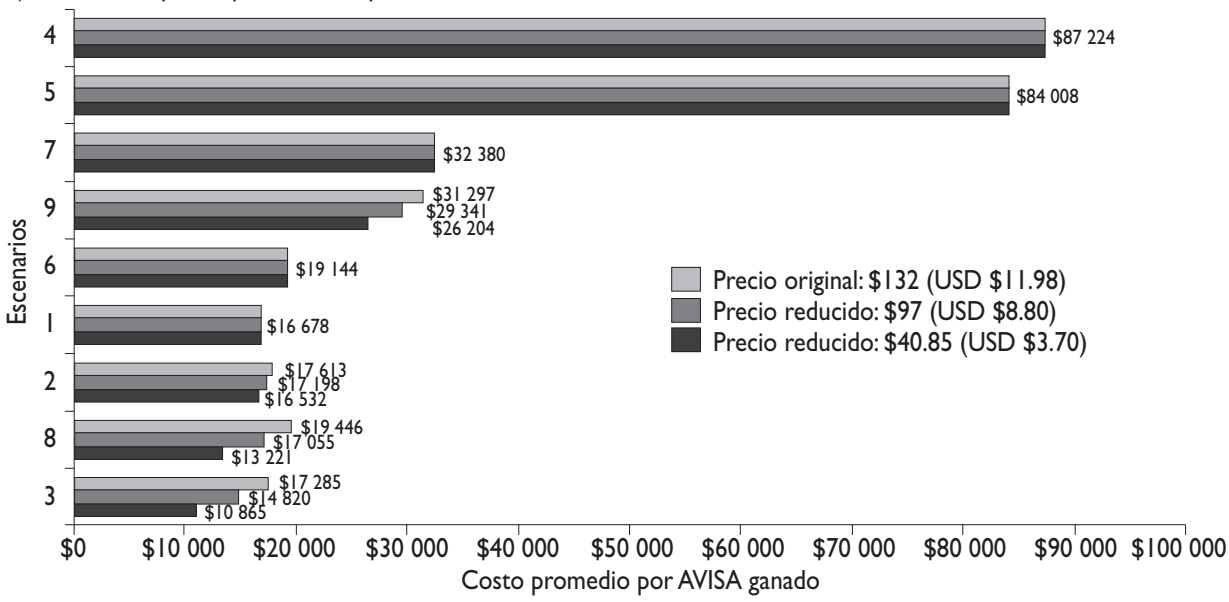

I: tamizaje con Papanicolaou según NOM (cobertura, 80\%); 2: tamizaje con Papanicolaou (cobertura, 80\%) y CH (cobertura, 10\%), ambos según NOM;3:tamizaje con $\mathrm{CH}$ (cobertura, 80\%); 4:vacunación (cobertura, I0\%; cohorte, niñas de I2 años); 5 :vacunación (cobertura, $80 \%$; cohorte, niñas de I2 años; 6 : vacunación (cobertura, 10\%; cohorte, niñas de 12 años) y tamizaje con Papanicolaou (mujeres, 25-64; cobertura, $80 \%$ ); 7:vacunación (cobertura, $80 \%$; cohorte, niñas de I2 años) y tamizaje con Papanicolaou (cobertura, 80\%); 8: vacunación (cobertura, 10\%; cohorte, niñas de 12 años) y tamizaje con CH (cobertura, $80 \%$ ); 9 : vacunación (cobertura, $80 \%$; cohorte, niñas de 12 años) y tamizaje con $\mathrm{CH}$ (cobertura, 80\%). Precio del Papanicolaou considerado: 86 pesos. Fuente: Unidad de Análisis Económico Figura 3. Análisis de sensibilidad de las variables más importantes del análisis de la relación costo-efectividad
generalizada para el CaCu en México 
combinación de las intervenciones más efectivas en cuanto al costo que mejoran el proceso de eficiencia distributiva de recursos financieros limitados en el contexto mexicano.

La principal ventaja del modelo desarrollado consiste en analizar de modo simultáneo la evolución tanto de las cohortes de niñas vacunadas como de las cohortes de mujeres tamizadas. La limitación más importante del modelo es atribuible a la falta de datos específicos para México en algunos parámetros epidemiológicos, sin que esta falta vulnere la consistencia del modelo o la validez de los resultados, ya que se lleva a cabo un análisis de sensibilidad.

Los resultados obtenidos del ACEG son consistentes con estudios similares notificados en otras publicaciones, ${ }^{*}, 16-20$ en las que se presentan resultados de la relación costo-efectividad para las tres intervenciones bajo diversos escenarios de cobertura, países y modelos de cohorte; de éstos destacan el papel del costo de la vacuna anti-VPH y la periodicidad del $\mathrm{CH}$ en países en desarrollo, como Brasil o Perú. 17,19,38

Los resultados para el tamizaje, en términos de la relación costo-efectividad, sugieren una mejoría del PNTCaCu en México al introducir una combinación selectiva de Papanicolaou y CH. Dicha introducción exige evaluar las ventajas comparativas de aplicación y confiabilidad de muestras obtenidas y analizadas en distintas poblaciones y áreas geográficas. ${ }^{30}$

Además, los resultados sugieren negociar un precio umbral basado en el análisis de sensibilidad para reducir el precio por prueba de $\mathrm{CH}$ en virtud del volumen de compra que una introducción gradual al sector público implicaría. ${ }^{\ddagger}$ Si se efectúa esta introducción hay que cuidar el mantenimiento de la cobertura en el plano nacional, que en la actualidad alcanza $80 \%$ de la población objetivo cada tres años. ${ }^{7}$

Los resultados obtenidos para la vacuna anti-VPH muestran que esta intervención genera un efecto finan-

\footnotetext{
* Hernandez-Avila M, Lazcano-Ponce E, Reynales-Shigematsu L, Rodriguez E, Myers E. Cost-effectiveness analysis of a potential human papillomavirus vaccine (HPV) and its epidemiological and economic impact on prevention and treatment of cervical cancer in Mexico, technical report, 2006. Merk Sharp and Dome, Dosier presentado al Consejo de Salubridad General para inclusión de la vacuna al Cuadro Básico de Medicamentos. Documento no publicado.

₹ Centro Nacional de Equidad de Género y Salud Reproductiva. Propuesta de mejora del programa de prevención y control del $\mathrm{CaCu}$. Secretaría de Salud, 2007. Documento no publicado.
}

ciero de consideración. El monto anual en 2007 para el escenario de vacunación universal equivale a $286 \%$ del presupuesto asignado por parte del Centro Nacional para la Salud de la Infancia y la Adolescencia (CENSIA) a la compra de las siete vacunas del esquema básico para niños menores de cinco años. ${ }^{*}, 39$ Con el precio propuesto de 883 pesos por dosis, la introducción de la vacuna anti-VPH al esquema básico implicaría incrementar en grado significativo el financiamiento para esa área y ello complicaría la obtención de financiamiento para las vacunas antineumocócica, antiinfluenza, antirrotavirus pentavalente acelular y antihepatitis B, incorporadas en fecha reciente. Además, los resultados indican que un plan piloto es menos efectivo en cuanto al costo que la cobertura universal. En consecuencia, es insostenible desde el punto de vista económico su introducción al sector público mexicano por el momento. ${ }^{38} \mathrm{Si}$ se considera que la vacuna puede ser un medio para generar equidad en el acceso a intervenciones preventivas contra el $\mathrm{CaCu},{ }^{27}$ se sugiere negociar un precio umbral de 181 pesos por dosis -cuando se compara la $\mathrm{CH}$ en términos del costo y la efectividad-como uno de varios componentes clave antes de considerar su introducción universal al sector público mexicano.

No debe soslayarse que antes de cualquier cambio en el plano público es necesario realizar un análisis más detallado de aspectos no financieros y discutir hasta obtener un consenso sectorial para determinar las adecuaciones necesarias a la NOM sobre el $\mathrm{CaCu}$ y las repercusiones operativas del posible cambio en la reorganización de recursos humanos, financieros y de infraestructura nacional. Las discusiones deben prever también las repercusiones éticas y sociales de las intervenciones analizadas. Los consensos y las decisiones alcanzados con todas sus limitaciones deben señalarse de manera explícita en un proceso justo de decisiones. ${ }^{40}$ Las implicaciones de dichos aspectos se analizarán en un trabajo futuro.

\section{Agradecimientos}

Los autores agradecen la participación de Mirella Loustalot del CNEGYSR, Rosa María Ceballos del CENETEC y la de tres revisores anónimos por sus enriquecedores comentarios.

\footnotetext{
* El presupuesto del CENSIA representa alrededor de la mitad del monto erogado para la cobertura del sector público; la otra mitad corresponde a las instituciones de seguridad social.
} 


\section{Referencias}

I. Secretaría de Salud. Principales causas de mortalidad en mujeres 2005. Disponible en: http://sinais.gob.mx. Consultado en abril de 2007.

2. Bosch X, Sanjosé S. Chapter I: Human papillomavirus and cervical cancer-burden and assessment of causality.J Nat Cancer Inst Monogr 2003;31.

3.Alliance for Cervical Cancer Prevention. Natural history of cervical cancer. Disponible en:www.path.org/files/RH_natural_history_of_cc_ fs.pdf. [Consultado abril 2007].

4. Dunne E. HPV vaccines. Disponible en: njlmn.rutgers.edu/cdr/docs/02HPVVaccines_Dunne.pdf. [Consultado abril 2007].

5. Subsecretaría de Promoción y Prevención de la Salud. NOM-0I4-SSA2 1994: Sobre el control y tratamiento del cáncer cérvico-uterino. México: Secretaría de Salud, Diario Oficial de la Federación, 3 I de mayo de 2007. 6. Instituto Nacional de Salud Pública.Autotoma vaginal en casa para identificación de ADN de VPH y su utilidad en la detección oportuna de cáncer cervical: una intervención en usuarias del Seguro Popular. México: Instituto Nacional de Salud Pública, 2006.

7. Centro Nacional de Equidad de Género y Salud Reproductiva. Informe de rendición de cuentas de la administración 2000-2006; etapa 3: informe que comprende del I de diciembre 2000 al 30 de noviembre 2006. México: Centro Nacional de Equidad de Género y Salud Reproductiva, 2007. 8. Subsecretaría de Promoción y Prevención de la Salud. Programa de acción: cáncer cérvico-uterino. México: Secretaría de Salud, 2002. 9. Lazcano-Ponce E, Castro R, Allen B, Najera P, Alonso de Ruiz P, Hernandez-Avila M. Barriers to early detection of cervical-uterine cancer in Mexico. JWomen Health 1999;8(3):399-408.

10. Secretaría de Salud. Sistema de protección social en salud: elementos conceptuales, financieros y operativos. 2a ed. México: Fondo de Cultura Económica, 2006

I I.Tan-Torres T, Baltussen R,Adam T, Hutubessy R,Acharya A, Evans D, et al. Making choices in health:WHO guide to cost-effectiveness analysis. Geneva:World Health Organization, 2003.

12. FUTURE II Study Group. Quadrivalent vaccine against human papillomavirus to prevent high-grade cervical lesions. N Engl J Med 2007:356:19.

13. Garnett G, Kim J, French K, Goldie S. Chapter 2I: Modelling the impact of HPV vaccines of cervical cancer and screening programmes. Vaccine 2006;24(S3): 178-186.

14. Goldie S, Kuhn L, Denny L, Pollack A, Wright T. Policy analysis of cervical cancer screening in low-resource settings, clinical benefits and cost-effectiveness. JAMA 200I;285:3107-3II5.

15. Goldie S, Kohli M, Grima D, Weinstein M, Wright T, Bosch F, Franco E. Projected clinical benefits and cost-effectiveness of a Human Papillomavirus 16/I8 vaccine.J Nat Cancer Inst 2004;96:8.

16. Goldie S, Kim J,Wright C. Cost-effectiveness of human papillomavirus DNA testing for cervical cancer screening in women aged 30 years or more.Am Coll Obstetr Gynecol 2004;103:4.

17. Goldie S, Gaffikin L, Goldhaber-Fiebert J, Gordillo-Tobar A, Levin $C$, Mahé $\mathrm{C}$, et al. Cost-effectiveness of cervical cancer screening in five developing countries. N Engl J Med 2005;353:2158-2168.

18. Goldie S, Kim J, Myers E. Chapter 19: Cost-effectiveness of cervical cancer screening.Vaccine 2006;24(suppl 3):164-170.

19. Goldie S, Kim J, Kobus K, Goldhaber-Fiebert J, Salomon J, O'shea

MK, et al. Cost-effectiveness of HPV I6, I8 vaccination in Brazil.Vaccine 2007;25:33.

20. Elbasha E, Dasbach E, Insigna R. Model for assessing human papillomavirus vaccination strategies. Emerg Infect Disease 2007;13:1.
21. Salmeron J, Lazcano-Ponce E, Lorincz A, Hernandez M, Hernandez P, Leyva A, et al. Comparison of HPV-based assays with Papanicolaou smears for cervical cancer screening in Morelos State, Mexico. Can Caus Cont 2003; I4(6):505-5I2.

22. Canadian Medical Association. Debate begins over public funding for HPV vaccine. Can Med Assoc J 2007; 176(7).

23.American Cancer Society.American Cancer Society Guideline for human papillomavirus (HPV) vaccine use to prevent cervical cancer and its precursors. American Cancer Society, 2007. Disponible en: caonline. amcancersoc.org/egi/content/full/57///7. Consultado en marzo de 2007. 24. Department of Health and Ageing. Australian Government funding of Gardasil.Australian Government: Department of Health and Ageing, 2006. 25. Markowitz L, Dunne E, Saraiya M, Lawson H, Chesson H, Unger E. Recommendations of the Advisory Committee on Immunization Practices (ACIP). MMWR Recomm Repor 2007;56(RR-2):I-24.

26. National Board of Health-Danish Centre for Health Technology Assessment. Reduction in the risk of cervical cancer by vaccination against human papillomavirus (HPV)-a health technology assessment, 2007. Disponible en: www.dacehta.dk/publications. Consultado en junio de 2007. 27. Organización Mundial de la Salud. Preparación de la introducción de las vacunas contra el virus del papiloma humano, orientaciones normativas y programáticas para los países. Ginebra: Organización Mundial de la Salud, 2006.

28. Sociedades Científicas de España.Vacunas profilácticas frente alVPH, documento de consenso de las sociedades cientíicas. Madrid: Sociedades Cientíícas de España, 2007.

29. Centers for Disease Control and Prevention. Cervical Cancer Screening Guidelines. Disponible en: www.cdc.gov/cancer/cervical. 30. Digene Corporation. HC2 HPV DNA Test, product insert. Disponible en: www.digene.com/healthcare/healthcare_products.html. Consultado en mayo de 2007.

3I. Flores S, Bishai F, Lazcano E, Shah P, Lorincz A, Hernandez M, Salmerón J. Improving cervical cancer screening in Mexico: results from the Morelos HPV Study. Salud Publica Mex 2003;45(suppl 3):S388-398.

32. Cox T, Cuzick J. HPV DNA testing in cervical cancer screening: from evidence to policies. Gynecol Oncol 2006;103:8-1I.

33. The University of Sheffield. Liquid-based citology in cervical screening: an updated rapid systematic review. Technology assessment report commissioned by the HTA Programme on behalf of The National Institute of Clinical Excellence. Sheffield:The University of Sheffield, 2003.

34. Consejo Nacional de Población. Proyecciones poblacionales 2005 2050. Disponible en: www.conapo.gob.mx. Consultado en mayo de 2007. 35. Cichon M, Newbrander W, Yamabana H,Weber A, Normand Ch, Dror $D$, et al. Modelling in health care finance. United Kingdom: International Labour Office and International Social Security Association, 1999.

36. González C, Rojas M, Hernández M, Oláiz G. Perfil del comportamiento sexual en adolescentes mexicanos de 12 a 19 años de edad: resultados de la ENSA 2000. Salud Publica Mex 2005;47(3). 37. Martínez J, Belmonte J, Gascueña M, Caballero LI, Sabroso C, García M. Cobertura e inmunorespuesta a la vacunación frente al virus de la Hepatitits B en adolescentes de la provincia de Guadalajara. Rev Esp Sal Pub 1998;72(2):119-126.

38. Agosti J, Goldie S. Introducing HPV vaccine in developing countries-key challenges and issues. N Engl J Med 2007;356(19).

39. Secretaría de Hacienda y Crédito Público. Cuenta de la Hacienda Pública Federal 2006. Disponible en: www.shcp.gob.mx. [Consultado junio 2007].

40. Daniels N, Sabin J. Setting limits fairly: can we learn to share medical resources? United Kingdom: Oxford University Press, 2002. 